\title{
Chaperons moléculaires et cytosquelette, des archébactéries aux mammifères
}

Nos connaissances progressent souvent de façon chaotique, ainsi une protéine majeure des testicules murins : Tcp-1 ( $T$-complex protein) serait un chaperon moléculaire comme l'a suggéré un travail récent sur les archébactéries [1].

TF55, une protéine de choc thermique de bactérie thermophile est apparentée à GroEL, protéine de choc thermique majeure d'E. coli

La plupart des organismes étudiés à ce jour réagissent à une élévation de température en synthétisant énergiquement une classe particulière de protéines : les protéines de choc thermique. Les archébactéries thermophiles ne font pas exception à cette règle. En particulier, Sulfolobus shibatae, dont la température de croissance optimale est autour de $70^{\circ} \mathrm{C}$ synthétise de grosses quantités d'une protéine de $55 \mathrm{kDa}$ : TF55, en réponse à une élévation de température à $85^{\circ} \mathrm{C}$. Cette protéine présente de faibles homologies de séquence en acides aminés et de très fortes ressemblances biochimiques avec la principale protéine de choc thermique des eubactéries: GroEL chez Escherichia coli, l'homologue de la Hsp60 des mycobactéries. Comme GroEL, TF55 est une protéine de $55 \mathrm{kDa}$ dont les molécules s'associent pour former des complexes stoechiométriques constitués de deux tores accolés et dont les coefficients de sédimentation sont de l'ordre de $20 \mathrm{~S}$. Ces complexes s'associent spécifiquement à des protéines dépliées dont ils empêchent l'agrégation. Ils possèdent une activité ATPase dépendant du magnésium. Or, on a montré que cette activité facilite la dissociation des complexes de GroEL avec les protéines dépliées. Chez E. coli, l'interaction réversible et modulée par l'ATP de met d'expliquer les fonctions multiples de cette protéine [2, 3] : catalyser l'assemblage d'édifices multiprotéiques, assister les chaînes polypeptidiques naissantes sur les ribosomes, atténuer les dénaturations provoquées par un choc thermique, corriger certaines mutations thermosensibles, faciliter la translocation de protéines vers l'extérieur des bactéries.

Chez les eucaryotes, on a trouvé il y a quelques années des protéines de choc thermique, les Hsp60, dont les séquences en amino-acides et les fonctions sont très semblables à celles de GroEL [2, 3]. Cependant, les Hsp60 eucaryotes, bien que codées par le génome nucléaire, sont exclusivement localisées dans les chloroplastes et les mitochondries. Or voilà que le clonage de TF55 dévoile un résultat surprenant: TF55 présente une très forte homologie avec d'autres protéines eucaryotes localisées dans le cytoplasme : les Tcp-1. Ce résultat confirme une hypothèse avancée sur la base de faibles homologies de séquences entre GroEL, Hsp60 et Tcp-1 [4] et a stimulé la réalisation d'expériences dont les résultats montrent que, d'une part, les protéines Tcp-1 forment des complexes toriques semblables à ceux de GroEL et de TF55, et que, d'autre part, Tcp-1 intervient au cours de la synthèse protéique et le repliement des protéines comme GroEL chez E. coli $[5,6]$.

Tcp-1, l'homologue murin de TF55, est une protéine abondante dans les spermatides

La protéine Tcp-1 a d'abord été caractérisée chez la souris, où elle est codée par un gène situé au milieu du complexe de gènes " $\mathrm{T}$ " sur le chromosome 11 , entre le centromère et les gènes $\mathrm{H}-2$ du complexe majeur d'histocompatibilité. Les souris hétérozygotes $T / t$ ont une queue courte $(\mathrm{T}=$ tail $)$. Les allèles $\mathrm{t}$ complets ou partiels sont généralement létaux à l'état homozygote $(t / t)$. Lee Silver et ses collaborateurs ont remarqué, en électrophorèse bidimensionnelle, que les souris possédant l'haplotype $t$ synthétisent des variants d'un groupe de huit protéines (Tcp de 1 à 8) [7]. L'expression de Tcp-1 chez la souris dépend fortement du développement ; elle est synthétisée dès le stade morula au troisième jour après la fécondation [8] ; c'est une protéine très abondante dans les testicules, en particulier dans les spermatides, sur la structure qui donne naissance à l'acrosome à partir de l'appareil de Golgi et sur les corps résiduels [9]. Tcp-1 est cependant absente dans les spermatozoïdes mûrs. Cette expression transitoire, associée à la biogenèse d'un organite, évoque la participation de GroEL à la formation de la capside du phage $\lambda$ ou la participation d'Hsp60 à l'assemblage des complexes multiprotéiques du chloroplaste et de la mitochondrie.

Comme GroEL, Tcp-1 pourrait aussi intervenir dans la synthèse protéique, c'est ce que pourrait suggérer l'analyse de l'haplotype $\mathrm{t}^{12}$, dont les embryons homozygotes dégénèrent au stade blastocyste, avant l'implantation dans l'utérus. En effet, en étudiant par électrophorèse bidimensionnelle les protéines synthétisées au stade 8-cellules, par des embryons individualisés, il a été observé que les embryons $\mathrm{t}^{12} / \mathrm{t}^{12}$ présentaient une synthèse défectueuse d'un groupe de protéines dont certaines sont identifiées et sont des constituants du cytosquelette [10]. La mutation t de la protéine Tcp-1 ne suffit pourtant pas, à elle seule, à conférer une létalité 
homozygote : en effet, les souris $\mathrm{t}^{\mathrm{w} 82} / \mathrm{t}^{\mathrm{w} 82}$ expriment le variant $\mathrm{Tcp}-1 \mathrm{~b}$ et sont viables. Cependant, il faut préciser que comme la plupart des chaperonines/protéines de choc thermique, GroEL coopère avec un auxiliaire, GroES [2, 3] ; or, les particules constituées de Tcp-1 sont constituées d'au moins sept polypeptides différents et semblent hétérogènes [5]. Le locus $\mathrm{T}$ s'étend sur $12 \mathrm{~cm}$, soit $0,75 \%$ du génome murin ; il se peut donc qu'un ou plusieurs variants de protéines coopérant avec Tcp-1 soient codés par des gènes $t$, identifiés ou non, et que seules certaines associations de variants $\mathrm{t}$ soient fonctionnellement déficientes.

Interactions entre les homologues eukaryotes de GroEL et le cytosquelette

Chez la souris, Tcp-1 est localisée dans le cytoplasme et sur la face cytoplasmique des structures trans de l'appareil de Golgi [9]. Dans le cytoplasme, Tcp-1 est associée à deux protéines de choc thermique Hsp70 et 4 à 6 protéines non identifiées au sein d'une particule hétéromérique de 800 à $950 \mathrm{kDa}$ [11]. La dynamique de l'appareil de Golgi est gouvernée par les microtubules. Chez la levure, on trouve un gène $T c p-1$ unique qui code pour une protéine qui semble interagir avec les microtubules [12]. Récemment, en cherchant à cloner des gènes codant pour des protéines interagissant avec les microtubules, le groupe de Claudine Rodrigues-Pousada a isolé le gène $T \mathrm{cp}-1$ chez Tetrahymena pyriformis (communication personnelle) et remarqué que les protéines Tcp-1 possédaient une séquence d'acides aminés, Proline-(Glycine), caractéristique des molécules favorisant la nucléation des microtubules [13]. Les protéines Tcp-1 pourraient donc, intervenir dans l'assemblage des microtubules, une hypothèse confirmée par l'observation de l'association de la tubuline néosynthétisée avec une particule de $900 \mathrm{kDa}$ dont le constituant majeur, un polypeptide de $58 \mathrm{kDa}$ est reconnu par un anticorps monoclonal anti-Tcp-1 [14]. Il semble que Hsp60 (l'homologue mitochondrial de GroEL apparentée à Tcp-1) puisse aussi interagir avec les microtubules en association avec une autre protéine de choc thermique cyto$\mathrm{m} / \mathrm{s} n^{\circ} 8$, vol. 8, octobre 92 plasmique de $70 \mathrm{kDa}, \mathrm{Hsc70}$ [15]. L'association de la Hsp60 avec les filaments intermédiaires (cytoplasmiques) a aussi été rapportée [16]. Enfin, l'interaction entre Tcp-1 et les microfilaments est plausible puisqu'un article récent vient de décrire la présence d'une protéine apparentée à $\mathrm{T} c \mathrm{p}-1$ dans un complexe multiprotéique toroïdal, isolé et caractérisé pour sa capacité à faciliter le repliement de la $\beta$ actine dénaturée avec hydrolyse d'ATP en présence de magnésium [17].

Les archébactéries posséderaient un cytosquelette et des protéines apparentées à l'actine et à la tubuline $[18$, $19]$; il n'est donc pas fortuit de trouver une meilleure homologie entre TF55 et Tcp-1 qu'entre GroEL des eubactéries et Tcp-1.

Ainsi, ces protéines apparentées contribueraient à la dynamique du cytosquelette : d'une part, elles favoriseraient la polymérisation des microfilaments, des microtubules et des filaments intermédiaires au voisinage de certains organites, et, d'autre part, elles assureraient l'ancrage de ces organites au cytosquelette

\section{Olivier Bensaude}

Groupe de biologie du stress cellulaire, génétique moléculaire, URA Cnrs 1302, Ecole normale supénieuse, 46, nue d'Ulm, 75230 Paris Cedex 05, France.

\section{RÉFÉRENCES}

1. Trent JD, Nimmersgern E, Wall JS, Hartl FU, Horwich AL. A molecular chaperone from a thermophilic archaebacterium is related to the eukaryotic protein t-complex polypeptide-1. Nature 1991; 354 : 490-3.

2. Gething MJ, Sambrook J. Protein folding in the cell. Nature 1992 ; $355: 33-45$.

3. Ellis RJ, Van der Vies SM. Molecular chaperones. Annu Rev Biochem 1991; 60: 321-47.

4. Gupta RS. Sequence and structural homology between a mouse T-complex protein Tcp-1 and the "chaperonin "family of bacterial (groEL, 60-65 kDa heat shock antigen) and eukaryotic proteins. Biochem Int 1990 $20: 833-41$

5. North G. A cytoplasmic chaperonin? Nature 1991 ; 354 : 434-5.
6. Ellis J. Cytosolic chaperonin confirmed. Nature $1992 ; 358$ : 191.

7. Silver L, Uman J, Danska J, Garrels JI. A diversified set of testicular proteins specified by genes within the mouse t complex. Cell $1983 ; 35: 35-45$.

8. Sanchez ER, Erickson RP. Expression of the $T c p-1$ locus of the mouse during early embryogenesis. J Embryol Exp Morphol 1985; 89 : 113-22.

9. Willinson $\mathrm{K}$. The $\mathrm{t}$ complex polypeptide 1 (Tcp-1) is associated with the cytoplasmic aspect of Golgi membranes. Cell 1989 ; 57 : 621-32.

10. Nozaki M, Iwakura $\mathrm{Y}$, Matsuhiro A. Studies of developmental abnormalities at the molecular level of mouse embryos homozygous for the $t^{12}$ lethal mutation. Dev Biol 1986 ; $113: 17-28$.

11. Lewis VA, Hynes GM, Zheng D, Saibil $\mathrm{H}$, Willison K. T-complex polypeptide-1 is a subunit of a heteromeric particle in the eukaryotic cytosol. Nature $1992 ; 358$ : 249-52.

12. Ursic D, Culbertson MR. The yeast homolog to mouse Tcp-1 affects microtubulemediated processes. Mol Cell Biol 1991; 11 : 2629-40.

13. Joly JC, Flynn G, Purich DL. The microtubule-binding fragment of microtubuleassociated protein-2 : location of the proteaseaccessible site and identification of an assembly-promoting peptide. J Cell Biol 1989 ; 109 : 2289-94.

14. Yaffe MB, Farr GV, Miklos D, Horwich A, Sternlich ML, Sternlich H. TCP 1 complex is a molecular chaperone in tubulin biogenesis. Nature 1992 ; 358 : 245-8.

15. Gupta R. Mitochondria, molecular chaperone proteins and the in vivo assembly of microtubules. Trends Biochem Sci 1990; 15 : 415-8.

16. Mose-Larsen P, Bravo R, Fey SJ, Small JV, Celis JE. Putative association of mitochondria with a subpopulation of intermediate filaments in cultured human skin fibroblasts. Cell 1982; $31:$ 681-92.

17. Gao Y, Thomas JO, Chow RL, Lee $\mathrm{GH}$, Cowan NJ. A cytoplasmic chaperonin that catalyzes $\beta$-actin folding. Cell $1992 ; 69$ : 1043-50.

18. Sioud M, Baldacci G, Forterre P, de Recondo AM. Antitumor drugs inhibit the growth of halophilic archaebacteria. Eur J Biochem $1987 ; 169$ : 231-6.

19. Searcy DG, Hixon WG. Cytoskeletal origins in sulfur-metabolizing archaebacteria. Biosystems $1991 ; 25: 1-11$.

\section{TIRÉS A PART}

O. Bensaude. 